\title{
Probing the quantum commutation rules through cavity QED
}

\author{
Qingqing Sun, ${ }^{1,2}$ M. Al-Amri, ${ }^{2,3}$ and M. Suhail Zubairy ${ }^{1,2}$ \\ ${ }^{1}$ Department of Physics and Institute of Quantum Studies, Texas A\&M University, College Station, Texas 77843-4242, USA \\ and Texas A\&M University at Qatar, Education City, P.O. Box 23874, Doha, Qatar \\ ${ }^{2}$ Max-Planck-Institut für Kernphysik, Saupfercheckweg 1, 69117 Heidelberg, Germany \\ ${ }^{3}$ The National Centre for Mathematics and Physics, KACST, P.O. Box 6086, Riyadh 11442, Saudi Arabia
}

(Received 26 June 2008; published 1 October 2008)

\begin{abstract}
We propose a cavity QED-based scheme to observe the noncommutativity between the creation and annihilation operators. By conditional measurement after the atom-field interaction in a cavity, it is possible to achieve a photon-added-then-subtracted state and a photon-subtracted-then-added state, which have different statistical properties. This scheme has the advantages of simple analytical results and much higher generation probabilities. We show that the ideal results of one-cycle photon addition and subtraction are approached in the short time limit. We also discuss the possible implementation scheme.
\end{abstract}

DOI: 10.1103/PhysRevA.78.043801

PACS number(s): 42.50.Dv, 03.65.Ta

\section{INTRODUCTION}

The commutation relation between the creation and annihilation operators $a^{\dagger}$ and $a$ has long been an intriguing part of quantum mechanics. It is the cause of zero point energy, including the vacuum fluctuation. Unlike classical systems where the order of adding and subtracting identical particles does not make any difference, in quantum systems each sequence gives a unique result due to the noncommutativity between $a^{\dagger}$ and $a$. A direct observation of these different results would be of fundamental interest.

With the progress of quantum state engineering, it is now possible to create a photon-added state or photon-subtracted state by conditional measurement. One way of doing this is through a beam splitter [1]. Dakna showed that if the initial state and a Fock state are injected at the two input channels, then the photon number counting of the output Fock state reduces the other output channel into a corresponding photon-added or photon-subtracted state [2]. Another method is through parametric amplification [3-5], where the photon counting at the idler mode could reduce the signal mode into a number state or a photon-added state [6]. Ban has shown the equivalence between the two methods [7].

Conditional measurement can also be applied to atomfield interaction in a cavity. For example, for an excited twolevel atom going through a cavity in the short interaction time limit, if the outgoing atom is detected to be in the ground state, then the field in the cavity will be reduced to the single photon-added state. The first proposal was made by Agarwal and Tara to produce the photon-added coherent state and it exhibits nonclassical properties [8]. Later came a series of discussions about various states after photon addition, such as photon-added squeezed vacuum states [9], even and odd coherent states [10], thermal states [11], and twomode squeezed vacuum states [12].

Recently Zavatta et al. realized the single photon addition for a coherent state [6], and showed the smooth transition between quantum (Fock) state and a classical (coherent) state by preparing different initial states. The same group achieved single photon addition and subtraction by using a setup involving a beam splitter and a parametric amplifier [13]. This provided a striking direct observation of the noncommutativity. They found that for a thermal state, counterintuitively a photon subtraction increases the average photon number. While the Wigner function for the photon-added-thensubtracted state has a positive dip at the center, the dip becomes negative for the photon-subtracted-then-added state.

These interesting results and their fundamental importance motivated us to conceive a similar experiment in cavity quantum electrodynamics (QED). There are certain advantages in such a scheme. The state generation probability is much higher as compared to the beam splitter setup. Our simple system allows an analytical form for the final density operator. The rapid development [14] in the field of cavity QED has provided all the necessary technology for the experimental implementation, as we will discuss in Sec. V.

\section{GENERAL THEORY}

In order to observe the noncommutativity between the creation and annihilation operators $a^{\dagger}$ and $a$, the most straightforward way is to compare the one-cycle photonadded-then-subtracted and photon-subtracted-then-added states. If the two sequences lead to different results then it is a clear sign of noncommutativity.

Ideally, a single photon addition to a state $|\Psi\rangle$ changes it into $a^{\dagger}|\Psi\rangle$. The density operator $\rho^{(0)}$ is correspondingly changed into $\sigma^{(a)}=N a^{\dagger} \rho^{(0)} a$ where $N$ means normalization of the density operator. If then followed by a single photon subtraction, the final density operator would be

$$
\sigma^{(s a)}=N a a^{\dagger} \rho^{(0)} a a^{\dagger} .
$$

This is the ideal result in which the photon only undergoes one cycle. The ideal density operator after one-cycle photonsubtraction-then-addition is

$$
\sigma^{(a s)}=N a^{\dagger} a \rho^{(0)} a^{\dagger} a .
$$

Due to the difficulty of deterministic single photon operations, one cannot obtain these ideal results exactly in experiment, but only approximate them.

Here we propose a cavity QED system for the experiment, in which an initial field is stored inside a cavity and two- 
level atoms are sent into the cavity one by one to interact with the field for some time before flying out. The atom-field interaction is governed by the unitary time-evolution operator [15]

$$
\begin{aligned}
U(t)= & \exp \left[-i g t\left(|a\rangle\left\langle b\left|a+a^{\dagger}\right| b\right\rangle\langle a|\right)\right] \\
= & \cos \left(g t \sqrt{a^{\dagger} a+1}\right)|a\rangle\left\langle a\left|+\cos \left(g t \sqrt{a^{\dagger} a}\right)\right| b\right\rangle\langle b| \\
& -i \frac{\sin \left(g t \sqrt{a^{\dagger} a+1}\right)}{\sqrt{a^{\dagger} a+1}} a|a\rangle\left\langle b\left|-i a^{\dagger} \frac{\sin \left(g t \sqrt{a^{\dagger} a+1}\right)}{\sqrt{a^{\dagger} a+1}}\right| b\right\rangle\langle a|,
\end{aligned}
$$

where $g$ is the atom-field coupling constant assumed to be real. The atom is detected when it flies out of the cavity. Only if it ends at the wanted level we claim a successful run, for which we measure the properties of the field left in the cavity. This postselection of atoms reduces the field in our desired way since they are entangled due to the interaction.

In the following sections we present the procedures and general results for the two-step detection and one-step detection, and show how the two-step results approach the ideal ones in the short time limit. These general formulas will be applied to the initial thermal state and coherent state in Secs. III and IV to observe the noncommutativity.

\section{A. Two-step detection}

In the two-step detection, we first send in an atom in the excited state $|a\rangle$ and detect it at the exit to select the ground state $|b\rangle$ result only. Then we send in another atom in the ground state and accept the case only if it ends in the excited state. When both conditions are satisfied, we measure the field left in the cavity.

The initial density operator of the system is $\rho^{(0)}|a\rangle\langle a|$. When the first atom is inside the cavity, the system evolves as $U(t) \rho^{(0)}|a\rangle\langle a| U^{\dagger}(t)$ if we ignore the damping for simplicity. Since we only choose the cases in which the atom leaves the cavity in the ground state after some interaction time $t$, the density operator of the field is reduced to

$$
\begin{aligned}
\rho^{(a)} & =N\left\langle b\left|U(t) \rho^{(0)}\right| a\right\rangle\left\langle a\left|U^{\dagger}(t)\right| b\right\rangle \\
& =N\left(a^{\dagger} \frac{\sin \left(g t \sqrt{a^{\dagger} a+1}\right)}{\sqrt{a^{\dagger} a+1}} \rho^{(0)} \frac{\sin \left(g t \sqrt{a^{\dagger} a+1}\right)}{\sqrt{a^{\dagger} a+1}} a\right) .
\end{aligned}
$$

Similarly for the second atom, the initial system is $\rho^{(a)}|b\rangle\langle b|$. After some interaction time $t^{\prime}$ and the atom detection, the final density operator of the field becomes

$$
\begin{aligned}
\rho^{(s a)} & =N\left\langle a\left|U\left(t^{\prime}\right) \rho^{(a)}\right| b\right\rangle\left\langle b\left|U^{\dagger}\left(t^{\prime}\right)\right| a\right\rangle \\
& =N\left(\frac{\sin \left(g t^{\prime} \sqrt{a^{\dagger} a+1}\right)}{\sqrt{a^{\dagger} a+1}} a \rho^{(a)} a^{\dagger} \frac{\sin \left(g t^{\prime} \sqrt{a^{\dagger} a+1}\right)}{\sqrt{a^{\dagger} a+1}}\right) .
\end{aligned}
$$

This is the photon-added-then-subtracted state after two-step detection.

The Taylor expansion of Eq. (5) yields

$$
\begin{aligned}
\rho^{(s a)}= & N\left(\left(g t^{\prime}\right)^{2} a \rho^{(a)} a^{\dagger}-\frac{\left(g t^{\prime}\right)^{6}}{3 !} a a^{\dagger} a \rho^{(a)} a^{\dagger} a a^{\dagger}+\cdots\right) \\
= & N\left(\left(g^{2} t t^{\prime}\right)^{2} a a^{\dagger} \rho^{(0)} a a^{\dagger}\right. \\
& \left.-\frac{\left(g^{4} t t^{\prime}\right)^{2}\left(t^{4}+t^{\prime 4}\right)}{3 !} a a^{\dagger} a a^{\dagger} \rho^{(0)} a a^{\dagger} a a^{\dagger}+\cdots\right) .
\end{aligned}
$$

In the short time limit when both $g t$ and $g t^{\prime}$ are very small, the one-cycle term is dominant so our result $\rho^{(s a)}$ approaches the ideal result $\sigma^{(s a)}$. With the increase of either interaction time, the two-cycle term comes into play and causes the deviation. Compared to Eq. (S5) of Ref. [13], where their parameters $\lambda$ and $\theta$ play the same roles as our $g t$ and $g t^{\prime}$, our expression is simpler and the unwanted terms start from higher order $(g t)^{6}$ instead of $\lambda^{4}$. This means they disappear much faster in the short time limit.

In the reversed order we first send in an atom in the ground state and only accept the results when it exits in the excited state. Then we send in another atom in the excited state and detect it in the ground state. The density operator of the photon-subtracted-then-added state $\rho^{(a s)}$ can be obtained from Eqs. (4) and (5) by simply switching levels $a$ and $b$.

\section{B. One-step detection}

A simpler setup is always preferable, so we also investigate the possibility of one-step detection. In pursuing the one-cycle photon-added-then-subtracted state, we send in an excited atom and detect it at the exit to choose the excited state result only. The field density operator is reduced to

$$
\rho_{1}^{(s a)}=N\left\langle a\left|U(t) \rho^{(0)}\right| a\right\rangle\left\langle a\left|U^{\dagger}(t)\right| a\right\rangle,
$$

where the subscript 1 means one step. Similarly we try to approximate the one-cycle photon-subtracted-then-added state by sending and detecting the atom in the ground state. The field density operator becomes

$$
\rho_{1}^{(a s)}=N\left\langle b\left|U(t) \rho^{(0)}\right| b\right\rangle\left\langle b\left|U^{\dagger}(t)\right| b\right\rangle .
$$

\section{INITIAL THERMAL STATE}

\section{A. Results of two-step detection}

In order to see the effect of these conditional measurements we consider an example of the thermal field. The initial density operator is

$$
\rho_{t h}=\sum_{n=0}^{\infty} \frac{\bar{n}^{n}}{(1+\bar{n})^{1+n}}|n\rangle\langle n|,
$$

where $\bar{n}$ is the average photon number of the thermal state. Using the formulas in Sec. II A we obtain the final density operators for the two sequences

$$
\rho^{(s a)}=\frac{\sum_{n=0}^{\infty} r^{n} \sin ^{2}(g t \sqrt{n+1}) \sin ^{2}\left(g t^{\prime} \sqrt{n+1}\right)|n\rangle\langle n|}{\sum_{n=0}^{\infty} r^{n} \sin ^{2}(g t \sqrt{n+1}) \sin ^{2}\left(g t^{\prime} \sqrt{n+1}\right)},
$$




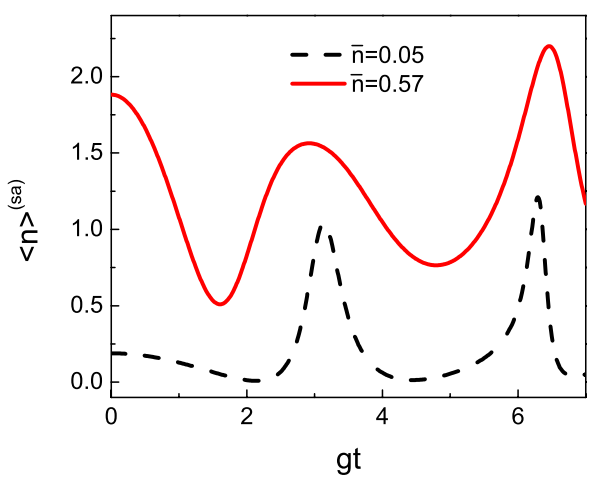

FIG. 1. (Color online) Average photon numbers $\langle n\rangle^{(s a)}$ change with the interaction time $g t$, while $g t^{\prime}$ is fixed at $0.1 \pi$. At most times they are larger than the initial photon number. The dips on the curves are caused by Rabi oscillations.

$$
\rho^{(a s)}=\frac{\sum_{n=1}^{\infty} r^{n} \sin ^{2}(g t \sqrt{n}) \sin ^{2}\left(g t^{\prime} \sqrt{n}\right)|n\rangle\langle n|}{\sum_{n=1}^{\infty} r^{n} \sin ^{2}(g t \sqrt{n}) \sin ^{2}\left(g t^{\prime} \sqrt{n}\right)},
$$

where $r=\bar{n} /(1+\bar{n})$. These simple analytical forms cannot be obtained in the beam splitter and/or parametric amplifier scheme. They allow us to analyze how the results change with the parameters, such as $\bar{n}$ and the interaction times.

With these density operators we can find any property of the final field. For example, we can prove the relation between the average photon numbers of the two sequences,

$$
\begin{aligned}
\langle n\rangle^{(s a)}+1= & \frac{\sum_{n=0}^{\infty} r^{n} \sin ^{2}(g t \sqrt{n+1}) \sin ^{2}\left(g t^{\prime} \sqrt{n+1}\right)(n+1)}{\sum_{n=0}^{\infty} r^{n} \sin ^{2}(g t \sqrt{n+1}) \sin ^{2}\left(g t^{\prime} \sqrt{n+1}\right)} \\
= & \frac{\sum_{n=0}^{\infty} r^{1+n} \sin ^{2}(g t \sqrt{n+1}) \sin ^{2}\left(g t^{\prime} \sqrt{n+1}\right)(n+1)}{\sum_{n=0}^{\infty} r^{1+n} \sin ^{2}(g t \sqrt{n+1}) \sin ^{2}\left(g t^{\prime} \sqrt{n+1}\right)} \\
= & \frac{\sum_{n=0}^{\infty} r^{n} \sin ^{2}(g t \sqrt{n}) \sin ^{2}\left(g t^{\prime} \sqrt{n}\right) n}{\sum_{n=0}^{\infty} r^{n} \sin ^{2}(g t \sqrt{n}) \sin ^{2}\left(g t^{\prime} \sqrt{n}\right)}=\langle n\rangle^{(a s)} .
\end{aligned}
$$

In deriving the above equation, we multiply both the numerator and the denominator with a factor $r$ in the second step, and reorganize the symbol $1+n$ into $n$ in the third step. Equation (11) clearly shows the noncommutativity between the creation and annihilation operators, valid for any interaction times.

In Fig. 1 we show how $\langle n\rangle^{(s a)}$ changes with the interaction time $g t$ at a fixed $g t^{\prime}=0.1 \pi$. For most of the $g t$, the average photon number after photon-addition-then-subtraction is

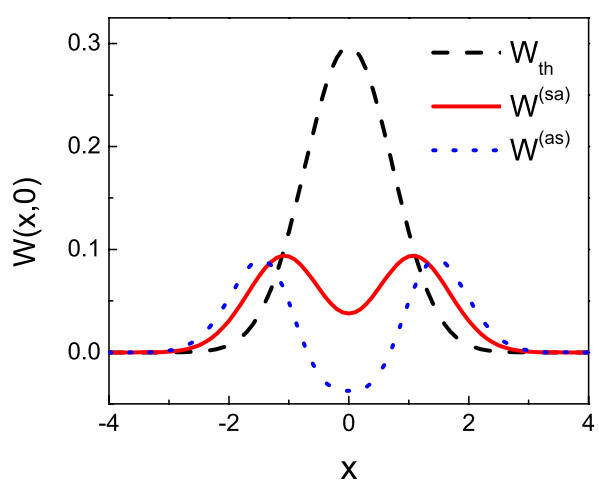

FIG. 2. (Color online) Wigner functions for the short interaction times $g t=g t^{\prime}=0.1 \pi$ and $\bar{n}=0.57$, which is very close to the ideal case of single photon addition and subtraction.

higher than the initial $\bar{n}$. This counterintuitive result, as explained in Ref. [13], is because the creation and annihilation operators produce a $\sqrt{n+1}$ (or $\sqrt{n}$ ) factor which increases the weight of higher number states in the final field. The dips and the peaks on these curves are caused by certain missing number states, as we will explain in the next section.

The generation probability $p^{(s a)}$ can be obtained from the normalization constant of $\rho^{(s a)}$,

$$
p^{(s a)}=\sum_{n=0}^{\infty} \frac{\bar{n}^{n}}{(1+\bar{n})^{1+n}} \sin ^{2}(g t \sqrt{n+1}) \sin ^{2}\left(g t^{\prime} \sqrt{n+1}\right) .
$$

We can similarly obtain $p^{(a s)}$ and prove their relation $p^{(a s)}$ $=r p^{(s a)}$. For $\bar{n}=0.57$ and close to short time limit $g t=g t^{\prime}$ $=0.1 \pi$, we have $p^{(s a)}=0.027$ and $p^{(a s)}=0.01$. This is over $10^{2}$ times larger than the generation probabilities in Ref. [13] at the fidelity level above $99 \%$. The reason is that we do not require very small $g t$ and $g t^{\prime}$ to eliminate the unwanted terms, since they start from higher order in our case.

We can also calculate the Wigner function from the density operators via [15]

$$
\begin{aligned}
W\left(\alpha, \alpha^{*}\right)= & \frac{2}{\pi^{2}} \exp \left(2|\alpha|^{2}\right) \int\langle-\beta|\rho| \beta\rangle \\
& \times \exp \left[-2\left(\beta \alpha^{*}-\beta^{*} \alpha\right)\right] d^{2} \beta,
\end{aligned}
$$

where $|\beta\rangle$ is a coherent state. For the initial thermal state the Wigner function can be shown to be

$$
W_{t h}\left(\alpha, \alpha^{*}\right)=\frac{2}{\pi(1+2 \bar{n})} \exp \left(-\frac{2|\alpha|^{2}}{1+2 \bar{n}}\right),
$$

which is positive everywhere in the phase space and peaks at the center, as shown in Fig. 2. The cycling of the photon dramatically changes the field. When both interaction times are small, the Wigner function after the photon-additionthen-subtraction process is also positive everywhere but has a dip at the center. For the reverse process, this central dip becomes negative. All these comply with the ideal situation and the experimental results from Ref. [13]. The different results from the two sequences reveal the noncommutativity between $a$ and $a^{\dagger}$. We show these Wigner functions only on 


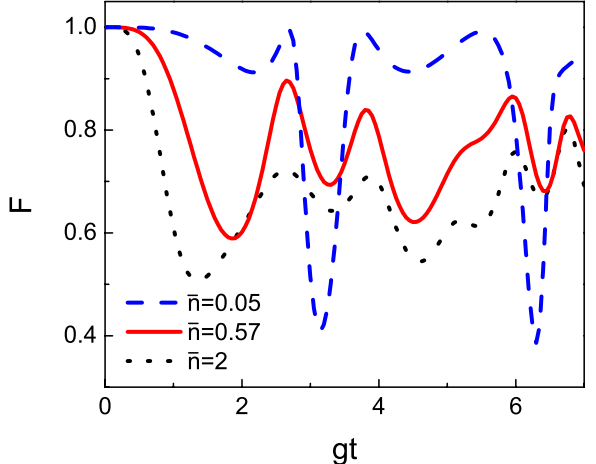

FIG. 3. (Color online) Two-step fidelity $\left(F^{(s a)} \equiv F^{(a s)}\right)$ with respect to time curves under different initial thermal states. We choose the same interaction times $g t=g t^{\prime}$. In the short time limit the fidelities go to 1 . The dips on the curves are caused by Rabi oscillations of different number states.

the $x$ axis because they are rotationally invariant for diagonal density operators.

\section{B. Fidelities of two-step detection}

In order to see how closely the final field $\rho$ simulates the ideal result $\sigma$, we calculate the fidelity. If the initial state is thermal, the ideal final density operators would be

$$
\begin{gathered}
\sigma^{(s a)}=\frac{\sum_{n=0}^{\infty} r^{n}(n+1)^{2}|n\rangle\langle n|}{\sum_{n=0}^{\infty} r^{n}(n+1)^{2}}, \\
\sigma^{(a s)}=\frac{\sum_{n=0}^{\infty} r^{n} n^{2}|n\rangle\langle n|}{\sum_{n=0}^{\infty} r^{n} n^{2}} .
\end{gathered}
$$

For two diagonal matrices $\rho=\Sigma_{n} \rho_{n}|n\rangle\langle n|$ and $\sigma=\Sigma_{n} \sigma_{n}|n\rangle\langle n|$, the fidelity is defined as $F=\Sigma_{n} \sqrt{\sigma_{n} \rho_{n}}$ [16]. By using the same trick as in Eq. (11) we can prove $F^{(s a)} \equiv F^{(a s)}$ in our system, for any interaction times.

The relation between the fidelities and the interaction times when $t=t^{\prime}$ is shown in Fig. 3. For all the different initial thermal states $\bar{n}=0.05,0.57$, and 2 , the fidelities approach 1 at small interaction times, which means we have an ideal result. The reason is simple, there is no time for multiple cycles and therefore it could only be single photon added or subtracted once in each step.

There are some interesting details in Fig. 3. All of these curves oscillate while they gradually decrease, and they all have many dips with some common ones at $g t=\pi, 2 \pi, \ldots$. The locations of these dips are $g t=k \pi / \sqrt{1+m}$ where $k$ $=1,2,3, \ldots$ and $m=0,1,2, \ldots$. At these interaction times, the number state $|m\rangle$ of the field recycles back and so does the atomic state; therefore the possibility of detecting the atom in the other state at the exit is essentially zero. This means $|m\rangle$

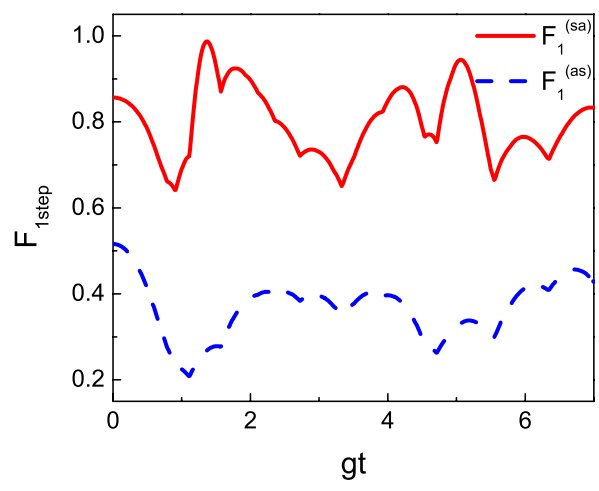

FIG. 4. (Color online) One-step fidelities for a thermal state with $\bar{n}=0.57 . F_{1}^{(s a)}$ can approach 1 at some later time, while $F_{1}^{(a s)}$ is always low.

does not pass its information to the field after postselection and causes the deviation dip. The depth of the dip mainly depends on the significance of $|m\rangle$ in the initial field. For $\bar{n}$ $=0.05$ the $|0\rangle$ state is the most important one and there is a small part of $|1\rangle$. So we see these deep dips at $\pi, 2 \pi$, and the shallow dips at $\pi / \sqrt{2}, 2 \pi / \sqrt{2}$. With the increase of $\bar{n}$ there are more significant number states and more dips. Some become so close together that they form a wide dip. An obvious example is the first dip of each curve.

\section{Fidelities of one-step detection}

Up to now we have been using two-step detection and found a good agreement with the ideal results in the short time limit. But how about one-step detection? The short time limit is no longer a good choice because the no-cycle term would be dominant, so we did not expect a good fidelity with respect to the ideal one-cycle result. However, for a moderate $\bar{n}=0.57$, the fidelity of one-step detection $F_{1}^{(s a)}$ rises up close to 1 at some interaction time, as shown in Fig. 4. The curve starts from below 1 as we expected. Some dips show up at locations $g t=(k+1 / 2) \pi / \sqrt{1+m}$ because the number state $|m\rangle$ is at half-cycle there and so the atom is in the ground state and gives no contribution to the detection. After all the significant number states (except for $|0\rangle$ whose factor caused by the operators is 1 and so its cycling makes no difference to the density operator) have passed the half-cycle points and before any of their one-and-a-half-cycle points, the detection gives a result close to the ideal one-cycle case. The result would be even better if we use a smaller $\bar{n}$.

On the contrary, if we send in an atom on the ground state and only consider the results when it exits on the ground state, the fidelity to the ideal photon-subtracted-then-added result $F_{1}^{(a s)}$ is quite low. The reason is that, for the ideal case, the $|0\rangle$ state would be eliminated by the annihilation operator, while it stays untouched in the one-step detection result.

\section{INITIAL COHERENT STATE}

We also want to see the results for other initial states. A natural choice is the coherent state $|\alpha\rangle$, which can be expanded into the number states 


$$
|\alpha\rangle=e^{-|\alpha|^{2} / 2} \sum_{n=0}^{\infty} \frac{\alpha^{n}}{\sqrt{n !}}|n\rangle .
$$

The ideal results for one-cycle photon addition and subtraction are

$$
\begin{gathered}
\sigma^{(a s)}=\left(\sum_{n=1}^{\infty} \frac{\alpha^{n}}{\sqrt{n !}} n|n\rangle \sum_{n^{\prime}=1}^{\infty} \frac{\alpha^{* n^{\prime}}}{\sqrt{n^{\prime} !}} n^{\prime}\left\langle n^{\prime}\right|\right) / \operatorname{Tr}, \\
\sigma^{(s a)}=\left(\sum_{n=0}^{\infty} \frac{\alpha^{n}}{\sqrt{n !}}(n+1)|n\rangle \sum_{n^{\prime}=0}^{\infty} \frac{\alpha^{* n^{\prime}}}{\sqrt{n^{\prime} !}}\left(n^{\prime}+1\right)\left\langle n^{\prime}\right|\right) / \operatorname{Tr},
\end{gathered}
$$

where the $\mathrm{Tr}$ in the denominator means the trace of the numerator. Here the average photon numbers have no fixed relation like Eq. (11) because $\rho_{n+1, n+1}^{(0)} / \rho_{n, n}^{(0)}$ is not a constant for all $n$.

In our system, after two-step detections, the field density operators become

$$
\begin{aligned}
\rho^{(a s)}= & \left(\sum_{n=1}^{\infty} \frac{\alpha^{n}}{\sqrt{n !}} \sin (g t \sqrt{n}) \sin \left(g t^{\prime} \sqrt{n}\right)|n\rangle\right. \\
& \left.\times \sum_{n^{\prime}=1}^{\infty} \frac{\alpha^{* n^{\prime}}}{\sqrt{n^{\prime} !}} \sin \left(g t \sqrt{n^{\prime}}\right) \sin \left(g t^{\prime} \sqrt{n^{\prime}}\right)\left\langle n^{\prime}\right|\right) / \mathrm{Tr}, \\
\rho^{(s a)=} & \left(\sum_{n=0}^{\infty} \frac{\alpha^{n}}{\sqrt{n !}} \sin (g t \sqrt{n+1}) \sin \left(g t^{\prime} \sqrt{n+1}\right)|n\rangle \sum_{n^{\prime}=0}^{\infty} \frac{\alpha^{* n^{\prime}}}{\sqrt{n^{\prime} !}}\right. \\
& \left.\times \sin \left(g t \sqrt{n^{\prime}+1}\right) \sin \left(g t^{\prime} \sqrt{n^{\prime}+1}\right)\left\langle n^{\prime}\right|\right) / \mathrm{Tr} .
\end{aligned}
$$

These reduce to the ideal result in the short time limit, as expected.

For the initial coherent state $|\alpha\rangle$ the Wigner function is a Gaussian function peaked at $\alpha^{\prime}=\alpha$,

$$
W_{\text {coh }}\left(\alpha^{\prime}, \alpha^{\prime *}\right)=\frac{2}{\pi} \exp \left(-2\left|\alpha^{\prime}-\alpha\right|^{2}\right) .
$$

For the ideal photon-subtracted-then-added state, the Wigner function has a negative dip located between the origin and $\alpha$, and a positive peak lying on their extension line. As an example we show the graph $W^{(a s)}$ in Fig. 5 when initially $|\alpha\rangle$ $=|1+i\rangle$. In the reversed order, the Wigner function $W^{(s a)}$ shown in Fig. 6 has similar features, although the negative dip is much shallower than the one for $W^{(a s)}$. We recall that in the ideal result for the thermal state, $W^{(s a)}$ is positive everywhere.

\section{IMPLEMENTATION}

The experimental realization of this scheme lies within the present technical limitation. Almost all aspects of the proposed experiment have been demonstrated in the experiments related to single atom interaction with field [17-21]

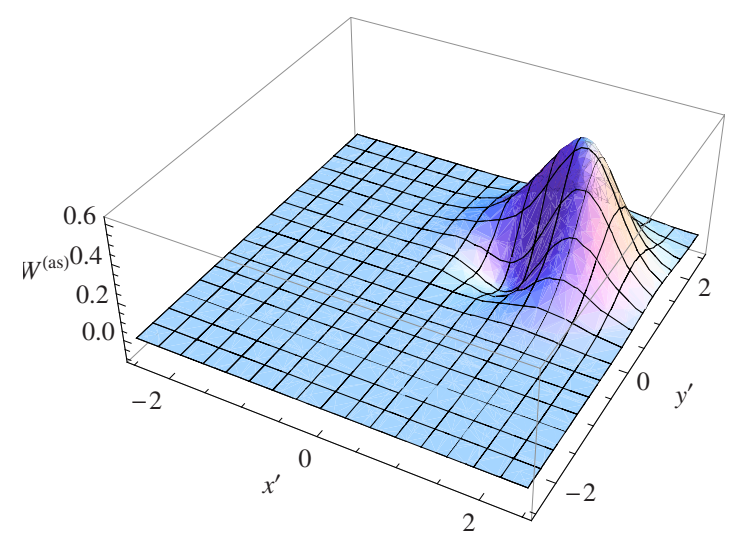

FIG. 5. (Color online) Ideal $W^{(a s)}$ when the initial state is a coherent state $|\alpha\rangle=|1+i\rangle$. Both the dip and the peak lie on the line connecting the origin and $\alpha$.

and quantum state measurement [22]. For example, we can use circular Rydberg atoms which behave like two-level atoms $[19,21]$. The levels $|a\rangle$ and $|b\rangle$ correspond to the Rydberg states with principal quantum number 51 and 50 , respectively. They are prepared one by one in a pulsed process $[23,24]$. The currently available high- $Q$ superconducting microwave cavity has been improved to $Q=4.6 \times 10^{9}$ and damping time $T_{c}=130 \mathrm{~ms}$ [25]. The initial thermal field can be prepared by cooling the system down to the desired temperature [17]. The initial coherent field can be injected into the cavity by a pulsed source $S$ [20]. The interaction time can be controlled by using atoms with different speeds [19]. To detect the atom level at the exit of the cavity, one can put an electric field outside to ionize and deflect the excited atoms [18].

The Wigner function of the final field can be detected without absorption via dispersive interaction [22]. Alternatively, we can measure the photon statistics and even the complete quantum state inside the cavity using a spectral technique [26].

\section{CONCLUSION}

In summary we have shown that a cavity QED experiment can be done to verify the commutation relation between

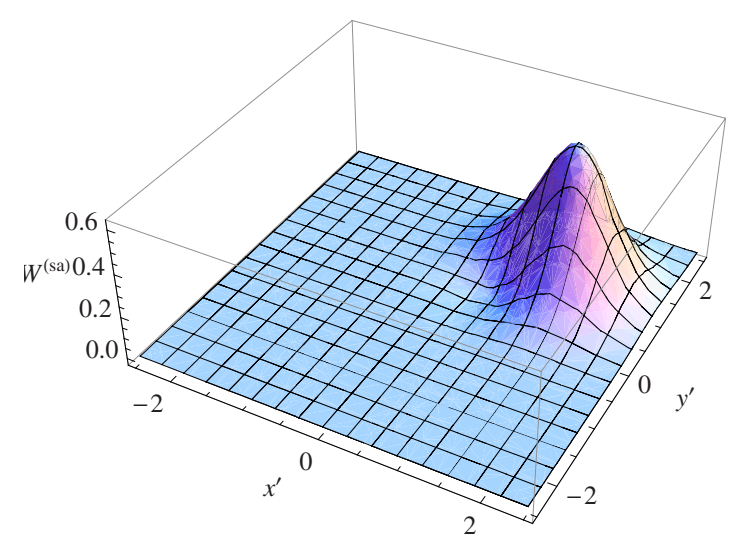

FIG. 6. (Color online) Ideal $W^{(s a)}$ when the initial state is a coherent state $|\alpha\rangle=|1+i\rangle$. Similar features as $W^{(a s)}$ but with a much shallower dip. 
the creation and annihilation operators. The proposed experiment gives a generation probability that is several orders of magnitude larger than the corresponding experiment using the beam splitter and parametric amplifier. In the short interaction time limit, two-step detection can provide an ideal photon-added-then-subtracted state or photon-subtractedthen-added state. Their different properties show the noncommutativity between $a^{\dagger}$ and $a$, which has its fundamental interest in quantum mechanics.

The simple analytical form of the final density operator allows us to see how the results change with the interaction times. Both the average photon numbers and the fidelity curves have many dips which can be explained by Rabi oscillations. Interestingly, we find even one-step detection is possible to generate a photon-added-then-subtracted state at some given time, when all the significant number states undergo one cycle.

The Wigner function for a coherent state also changes after photon addition and subtraction. The initial Gaussian peak becomes a positive peak and a negative dip on the line between the origin point and the initial center. The values and locations for these extreme points are different for the two alternated sequences. An interesting difference from the thermal state is that $W^{(s a)}$ can be negative now even in the ideal result.

In all these calculations we ignore the atomic decoherence and field damping because the interaction times are small compared to the long radiative lifetimes of the circular Rydberg states and the long damping time in the high- $Q$ cavity.

\section{ACKNOWLEDGMENTS}

One of us (M.S.Z.) would like to thank Hai-Woong Lee for helpful discussions. This research is supported by grants from the King Abdul Aziz City for Science and Technology (KACST) and the Qatar National Research Fund (QNRF). M.S.Z. would also like to thank the Alexander von Humboldt Foundation for supporting this research.
[1] M. Ban, J. Mod. Opt. 43, 1281 (1996).

[2] M. Dakna, L. Knll, and D.-G. Welsch, Eur. Phys. J. D 3, 295 (1998).

[3] Y. Yamamoto, S. Machida, M. Imoto, M. Kitagawa, and G. Björk, J. Opt. Soc. Am. B 4, 1645 (1987).

[4] B. Yurke and D. Stoler, Phys. Rev. A 36, 1955 (1987).

[5] G. S. Agarwal, Quantum Opt. 2, 1 (1990).

[6] A. Zavatta, S. Viciani, and M. Bellini, Science 306, 660 (2004).

[7] M. Ban, Opt. Commun. 143, 225 (1997).

[8] G. S. Agarwal and K. Tara, Phys. Rev. A 43, 492 (1991).

[9] Z. Zhang and H. Fan, Phys. Lett. A 165, 14 (1992).

[10] V. V. Dodonov, Y. A. Korennoy, V. I. Man'ko, and Y. A. Moukhin, Quantum Semiclassic. Opt. 8, 413 (1996).

[11] G. N. Jones, J. Haight, and C. T. Lee, Quantum Semiclassic. Opt. 9, 411 (1997).

[12] H. Lu, Phys. Lett. A 264, 265 (1999).

[13] V. Parigi, A. Zavatta, M. Kim, and M. Bellini, Science 317, 1890 (2007).

[14] H. Walther, B. T. H. Varcoe, B.-G. Englert, and T. Becker, Rep. Prog. Phys. 69, 1325 (2006).

[15] M. O. Scully and M. S. Zubairy, Quantum Optics (Cambridge University Press, New York, 1997).

[16] M. A. Nielsen and I. L. Chuang, Quantum Computation and Quantum Information (Cambridge University Press, Cambridge, 2000).
[17] G. Rempe, H. Walther, and N. Klein, Phys. Rev. Lett. 58, 353 (1987).

[18] J. Krause, M. O. Scully, and H. Walther, Phys. Rev. A 36, 4547 (1987).

[19] M. Brune, F. Schmidt-Kaler, A. Maali, J. Dreyer, E. Hagley, J. M. Raimond, and S. Haroche, Phys. Rev. Lett. 76, 1800 (1996).

[20] M. Brune, E. Hagley, J. Dreyer, X. Maitre, A. Maali, C. Wunderlich, J. M. Raimond, and S. Haroche, Phys. Rev. Lett. 77, 4887 (1996).

[21] S. Haroche, M. Brune, and J. M. Raimond, J. Mod. Opt. 54, 2101 (2007).

[22] L. G. Lutterbach and L. Davidovich, Phys. Rev. Lett. 78, 2547 (1997); G. Nogues, A. Rauschenbeutel, S. Osnaghi, P. Bertet, M. Brune, J. M. Raimond, S. Haroche, L. G. Lutterbach, and L. Davidovich, Phys. Rev. A 62, 054101 (2000).

[23] J. M. Raimond, M. Brune, and S. Haroche, Rev. Mod. Phys. 73, 565 (2001).

[24] S. Haroche and J. M. Raimond, Exploring the Quantum (Oxford University Press, Oxford, 2006).

[25] S. Kuhr, S. Gleyzes, C. Guerlin, J. Bernu, U. B. Hoff, S. Deléglise, S. Osnaghi, M. Brune, J.-M. Raimond, S. Haroche, E. Jacques, P. Bosland, and B. Visentin, Appl. Phys. Lett. 90, 164101 (2007).

[26] M. S. Zubairy, Phys. Lett. A 222, 91 (1996); Phys. Rev. A 57, 2066 (1998). 